\title{
Sobre el racismo, su negación, y las consecuencias para una educación anti-racista
}

\section{en la enseñanza secundaria chilena}

\author{
Andrea Riedemann \\ Universidad Alberto Hurtado, Santiago, Chile. \\ Email: ariedemann@uahurtado.cl \\ Carolina Stefoni \\ Universidad Alberto Hurtado, Santiago, Chile. \\ Email: cstefoni@uahurtado.cl
}

\begin{abstract}
Resumen $^{1}$ : En base a resultados preliminares de una investigación acerca de las maneras en que los colegios en Chile se están adaptando a la llegada de alumnos inmigrantes, este artículo se enfoca en el racismo presente en un liceo de enseñanza media, ubicado en la capital de Chile, y que se caracteriza por una alta presencia de alumnos afrodescendientes. A través de entrevistas y focus groups fue posible pesquisar una serie de situaciones que denotan visiones, discursos o prácticas racistas por parte de diferentes miembros de la comunidad educativa. El análisis de un breve cuestionario escrito, respondido por los alumnos haitianos del establecimiento confirma dicho diagnóstico. La mayor parte de la comunidad educativa tiende a negar, a través de diversos mecanismos, que exista racismo al interior del liceo. Los hallazgos de la investigación le plantean varios desafíos a una educación anti-racista, los que son discutidos en la parte final del artículo.

Palabras clave: inmigración haitiana, racismo en Chile, negación del racismo, educación anti-racista.
\end{abstract}

\section{On racism, its denial, and the consequences for an anti-racist education in chilean high school}

\begin{abstract}
Based on preliminary results of a research about the ways chilean schools are adapting to the growing amount of immigrant pupils, the article focuses on the actual racism present in a high school located in the country's capital and which is characterized by a high presence of pupils of african descent. Through interviews and focus groups it was possible to identify several situations denoting racist views, discourses or practices. The analysis of a short written questionnaire answered by the Haitian students confirms that diagnosis. The bigger part of this school community tends to deny the presence of racism in its school, and does so through various strategies. The findings of this research confronts the design of an anti-racist education in Chile with several challenges, which are discussed in the latter part of this article.
\end{abstract}

Keywords: Haitian immigration, racism in Chile, denial of racism, antiracist education. 


\section{Sobre o racismo, sua negação e as consequências para uma educação anti-racistano ensino secundáriochileno}

Resumo: Com base nos resultados preliminares de uma pesquisa sobre as maneiras pelas quais as escolas no Chile estão se adaptando à chegada de estudantes imigrantes, este artigo centra-se sobre o racismo presente na escola no ensino médio, localizado na capital de Chile, e que se caracteriza por uma elevada presença de alunos de afrodescendentes. Por meio de entrevistas e grupos focais foi possível pesquisar uma série de situações que denotam visões, discursos ou práticas racistas por diferentes membros da comunidade educativa. A análise de um breve questionário escrito,respondido pelos alunos do estabelecimento haitiano confirma o diagnóstico. A maior parte da comunidade educativa tende a negar, através de diversos mecanismos, que o racismo existe dentro da escola. Os resultados da pesquisa representam vários desafios para erguer uma educação anti-racista, os quais são discutidos na parte final do artigo.

Palavras-chave: Imigração do Haiti, racismo no Chile, negação do racismo, educação anti-racista.

$* * *$

\section{Introducción}

Han pasado cerca de veinte años desde que se inició en Chile su fenómeno inmigratorio más reciente, y existe cierta evidencia acerca de que en las escuelas chilenas cotidianamente los niños y jóvenes inmigrantes están expuestos a sufrir diversas experiencias de discriminación y racismo (Cortez, Loredo, Muñoz, Rodríguez, \& Vásquez, 2004; Pavez, 2012; Stefoni, Acosta, Gaymer, \& Casas-Cordero, 2008; Tijoux, 2013). Desde el ámbito académico lo anterior ha llevado, entre otras iniciativas, a la primera formulación de propuestas de política pública para una educación anti-racista, que junto a otros aspectos releva que al interior de las instituciones educativas se pueda "comprender que el racismo tiene una raigambre histórica que requiere ser reconocida, discutida y analizada para poder contribuir a la conformación de una sociedad chilena más justa" (Universidad de Chile, 2015, p. 10). La literatura sobre el racismo ha mostrado que en efecto éste no sólo tiene una raíz histórica (Geulen, 2007; Mosse, 2006), sino que adquiere formas diversas dependiendo de los diferentes tiempos y espacios en los que se manifiesta (Wieviorka, 2009, pp. 14-16). Bajo el entendido de que una iniciativa de educación anti-racista es en efecto necesaria en la sociedad chilena actual, y que requiere de una profunda comprensión acerca de cómo opera el racismo en este tiempo y espacio particular, el presente artículo pretende ser un aporte a la comprensión del racismo que actualmente se manifiesta en la enseñanza secundaria en Chile cuando su alumnado cuenta con jóvenes afrodescendientes, y específicamente proveniente de Haití. Como se verá en detalle más adelante, uno de los hallazgos más llamativos del caso en el cual se basa el presente artículo, es que junto a las diferentes situaciones de racismo que pudimos identificar, encontramos que la mayor parte de la comunidad educativa del liceo en cuestiónniega la existencia de racismo al interior del establecimiento. 
En términos de estructura, este artículo presenta en primer lugar información sobre la migración haitiana en Chile y datos proporcionados por el Mineduc acerca de la matrícula de niños y jóvenes haitianos en el sistema educativo nacional. A continuación se presenta el marco teórico y metodológico del estudio, y luego los principales hallazgos, divididos en dos partes: en la primera presentamos las principales situaciones de racismo que pudimos identificar en base a los relatos de los participantes del estudio, y en la segunda parte presentamos las estrategias a través de las cuales la comunidad educativa está negando la existencia de racismo en el establecimiento. Finalmente, presentamos una discusión sobre los aportes que la evidencia empírica recopilada le entrega a una reflexión sobre los desafíos de una educación anti-racista pertinente y contextualizada al caso de Chile.

\section{Migración haitiana y alumnos haitianos matriculados en establecimientos educativos chilenos}

“Aunque Haití fue el primer país de América Latina y el Caribe en independizarse y expulsar las colonias europeas de su territorio en $1804-$ apenas veinte años después de que lo hizo Estados Unidos- su población ha sufrido, a lo largo de su historia, las consecuencias de la violencia política, la precariedad económica, los desastres políticos y naturales y el intervencionismo político y económico”(Ceja Cárdenas, 2015, p. 2). Durante el siglo XIX y principios del XX, muchos haitianos migraron a enclaves del Caribe, particularmente Cuba y República Dominicana, para trabajar como jornaleros temporales en la cosecha (OIM-FLACSO, 2004, p. 12). Con la caída de la industria azucarera, los haitianos empezaron a migrar a Estados Unidos, Canadá, Francia y las colonias francesas en el Caribe. Durante el siglo XX aumentaron las migraciones hacia los Estados Unidos, con una primera ola migratoria impulsada por la ocupación norteamericana en la isla y una segunda ola migratoria desencadenada por la toma de poder de Duvalier (Ceja Cárdenas, 2015, p. 3). "Haití tiene una larga historia migratoria, sin embargo, a raíz del terremoto ocurrido en enero de 2010 y con los cambios en las políticas migratorias en Brasil y Ecuador, los flujos migrantes haitianos han ido transformándose, reconfigurando los destinos migratorios y dibujando nuevas rutas en el mapa latinoamericano [...]. Con el terremoto de 2010, según cifras oficiales, más de 200.000 personas murieron y más de 300.000 quedaron heridas, sin casa y obligadas a vivir en condiciones insalubres y de fuerte vulnerabilidad [...] lo que contribuyó a que siguiera aumentando el éxodo de la población fuera de Haití" (Ceja Cárdenas, 2015, pp. 2-3).

La investigación acerca de la migración haitiana en Chile hasta la fecha aún es muy acotada, y ha versado sobre temas urbanísticos (Castillo \& del Castillo, 2014), laborales (Valenzuela et al., 2014)y la "frontera imaginaria” que separa a los haitianos de Chile (Pérez Cosgaya, 2008). De acuerdo a la información que en su momento pudo recabar Teresa Pérez Cosgaya, en el año 2008 vivían en Chile alrededor de 100 personas haitianas (2008, p. 
73). Por su parte, los autores del estudio sobre temas urbanos determinaron, en base a la consulta a diferentes organismos, que en el año 2011 había en Chile cerca de 3.000 haitianos (Castillo \& del Castillo, 2014, p. 157). Por su parte, el ministro consejero de la embajada de Haití en Chile, en una entrevista concedida en mayo de 2014, planteaba que en esa fecha vivían más de 4.000 haitianos en Chile ${ }^{2}$. Esto confirma el planteamiento de que de manera posterior al terremoto de 2010 la emigración haitiana aumentó, y como ha sido evidente, parte de ella eligió a Chile, y en particular Santiago, como destino migratorio.

Los movimientos de personas suponen el arribo de hijos y familiares, quienes son parte central del proyecto migratorio. El acceso a la educación, salud y vivienda por parte de los y las trabajadores migrantes, así como de sus hijos y familiares, deja al descubierto los limites institucionales, sociales y culturales que tiene la implementación de una noción de igualdad de derechos en la sociedad chilena, evidenciando discursos que legitiman la desigualdad y el acceso condicionado a derechos, en base a argumentos racistas y xenófobos. Nos detendremos en la experiencia de un liceo de la Región Metropolitana, para mostrar y analizar cómo se hace presente la ideología racista.

De acuerdo a información proporcionada por la Unidad de Estadísticas del Ministerio de Educación ${ }^{3}$, en el año 2014 se registró una matrícula total de 245 alumnos en el sistema escolar chileno. La siguiente Tabla $\mathrm{N}^{\circ} 1$, que abarca desde la educación parvularia hasta la enseñanza media (incluyendo educación para adultos) muestra la distribución de ese total por nivel:

Tabla Nº1 Matrícula haitianos según Nivel y Dep. Adm., Año 2014

\begin{tabular}{|c|c|c|c|c|}
\hline Grados & Municipal & Part. Subv. & Part. Privado & Total \\
\hline Educación Parvularia & 29 & 9 & 0 & 38 \\
\hline Básica Regular & 105 & 51 & 2 & 158 \\
\hline Básica Adultos & 1 & 0 & 0 & 1 \\
\hline Educación Especial & 0 & 5 & 0 & 5 \\
\hline Media Regular & 22 & 13 & 0 & 35 \\
\hline Media Adultos & 5 & 3 & 0 & 8 \\
\hline Total & 162 & 81 & 2 & 245 \\
\hline
\end{tabular}

Fuente: Unidad de Estadísticas, Centro de Estudios, División de Planificación y Presupuesto, Ministerio de Educación

Como es posible apreciar en la tabla, en el año 2014 la mayor parte de la matrícula haitiana se encontraba en la enseñanza básica (64,5\%), mientras que en la enseñanza media se encontraba sólo el 14,3\% de la matrícula de alumnos haitianos en Chile. De acuerdo a información recabada durante el 
trabajo de campo, durante el presente año 2015 ha llegado una cantidad aún indeterminada, pero significativa, de alumnos haitianos nuevos a los establecimientos educativos chilenos.

Fuera de la Región Metropolitana, sólo se registran alumnos matriculados en las comunas de Coquimbo, IV Región (3 alumnos), Calera, V Región (2 alumnos) y Rancagua, VI Región (1 alumno). Dentro de la Región Metropolitana, las comunas de mayor matrícula haitiana son, en orden decreciente, Quilicura (81 alumnos), Estación Central (39), Pedro Aguirre Cerda (34), San Bernardo (18) y Cerrillos (15).

\section{Marco teórico y metodológico}

Son tres los conceptos centrales que requieren ser definidos en el marco del presente artículo: racismo, negación del racismo y educación anti-racista.

Entenderemos el racismo combinando un enfoque general con una definición más bien específica: en primer lugar, consideraremos como racismo cualquier expresión verbal o acción en la que se manifieste la creencia de que es válida la clasificación de los seres humanos en "razas" ${ }^{4}$ En segundo lugar, nos basaremos en la definición proporcionada por Albert Memmi acerca de la manera en que funciona el racismo: "El racismo consiste en la valoración de diferencias biológicas, sean estas reales o imaginarias, en beneficio de quien hace la definición y en desmedro de quien es sujeto de esa definición, con el fin de justificar hostilidad (social o física) y agresión” (Memmi, 2000). La distinción y posterior clasificación de seres humanos en estas categorías, es una construcción social que es parte de una ideología que busca convencer de la existencia real de las razas y de su organización en un sistema jerárquico 'natural'. Una situación que denota racismo será entonces cualquier situación en la que, en base a una diferencia entre quien observa y quien es objeto de esa observación, la diferencia es utilizada para inferiorizar al segundo, sea esto a través de un gesto, una expresión verbal (que puede oscilar desde una supuesta broma hasta una expresión directamente insultante) o una acción física. Adicionalmente -y esto es importante sobre todo con miras al objetivo de reflexionar sobre los desafíos que la actual realidad escolar le presenta a una educación anti-racista pertinente al contexto local- resulta relevante distinguir entre el así llamado racismo “antiguo”, científico o biológico, y el "nuevo” racismo (Barker, 1981), de tipo cultural o diferencialista (Taguieff, 2001). Uno de los elementos centrales que está a la base de la transición entre el racismo biológico y el racismo cultural, es que en el primero juega un rol muy relevante la creencia de que existen razas humanas -supuestamente reconocibles sobre todo a través de las diferencias corporales- mientras que en el segundo, el racismo se aleja del concepto más bien estático de raza, para enfocarse mayormente en las diferencias culturales. Tal como plantea en sus tesis Antonia Nguyen Vo, "generalmente, una acusación de racismo se asocia a comportamientos o actitudes explícitamente discriminatorios; suele ser reconocido cuando se 
manifiesta en agresiones físicas, explotación, expulsiones de inmigrantes o insultos explícitos. Sin embargo, existen muchas más manifestaciones de este fenómeno que no son tan obviamente reconocibles, como lo son las formas del llamado "nuevo racismo" y que, como consecuencia, son negadas” (Nguyen Vo, 2009, p. 1). Por último, es importante destacar que desde la educación anti-racista y otras corrientes el racismo se entiende eminentemente como una práctica discursiva, en la cual se expresa un determinado poder discursivo (Gillborn 2000, citado en: Auernheimer, 2003, p. 153). Como se verá en la siguiente sección, el material sobre el cual se basa el análisis del presente artículo fue recolectado a través de entrevistas y focusgroups, que son, por definición, prácticas discursivas. La consideración del racismo como una práctica discursiva junto al hecho de que el material recopilado en esta investigación es de tipo discursivo nos han llevado a incorporar algunos aportes del Análisis Crítico del Discurso al análisis y la interpretación de los datos recogidos.

Lo anterior nos vincula directamente al segundo concepto relevante en el marco del presente artículo:de acuerdo a los aportes del Análisis Crítico del Discurso, la negación del racismo es una de las características centrales del racismo contemporáneo (van Dijk, 1992, p. 87). Esta negación puede ser definida como "un mecanismo de defensa utilizado para salvaguardar la propia imagen positiva ante una amenaza que pueda dañarla” (Nguyen Vo, 2009, p. 27). Dicha amenaza por lo general está dada por una acusación explícita o implícita de racismo, y la defensa de la persona interpelada muchas veces se da a través de una negación. La negación también da cuenta de los procesos de invisibilización, naturalización y legitimación de un sistema de estratificación en base a categorías racializadas socialmente construidas. Con miras a los tópicos más recurrentes que emergieron en las entrevistas y focusgroups, a continuación se exponen algunas estrategias de negación, recopiladas a partir de diferentes documentos relativos al análisis del racismo por parte del Análisis Crítico del Discurso.

Una primera estrategia es la que podría denominarse como negación directa, o negación propiamente tal: en esta estrategia, se rechaza la acusación implícita o explícita de racismo sin mayores explicaciones. Una segunda estrategia es la de argumentar que no hubo intención de actuar de manera racista. El tema de la intencionalidad, y como contraparte, la falta de intención, es central en las estrategias de defensa, ya que en la práctica es muy difícil poder demostrar que una persona actuó con una intención racista (van Dijk, 1992, p. 92). Dentro de esta estrategia destacan las “bromas”, que junto a los "chistes" y burlas han sido definidos como "discursos no serios”, que tienen dos cualidades fundamentales: "por un lado, revelan prejuicios y estereotipos existentes en la sociedad, y, por otro, los reproducen en tanto contribuyen a su consolidación y favorecen su amplia y rápida circulación” (Edelstein, 1999, p. 265). Una tercera estrategia es la mitigación, que se refiere a bajarle el perfil a una situación, minimizarla, usar eufemismos o intentar dar una justificación a un discurso o una acción racista. La minimización puede ocurrir, entre otros, a través de la calificación de las situaciones racistas como incidentes aislados o de responsabilidades indi- 
viduales y acotadas (y no de responsabilidad colectiva o institucional). En relación al uso de eufemismos, típicamente aquellos actos que innegablemente son racistas, tienden a ser descritos en términos de discriminación, prejuicio, estereotipos, racialmente motivados, pero no como racistas (van Dijk, 1992, p. 93). La justificación, por su parte, se basa en la creencia de que si uno tiene un "buen" argumento, entonces la acción racista se verá legitimada. Finalmente, una cuarta estrategia de negación que presentamos aquí es la de revertir la acusación: la persona que acusa a otra de ser racista es, a su vez, acusada de racismo invertido hacia los blancos, de ser demasiado sensible y exagerada, de ser intolerante o de "ver racismo donde no lo hay" (van Dijk, 1992, p. 90).

A través de estos discursos de negación se observa el uso de referencias biologicistas y culturales para marcar distinciones y darle una condición de realidad que naturaliza y justifica, a ojos de los entrevistados y de la escuela como institución, la posición subalterna en la que son situados los hijos de migrantes haitianos.

En relación a la negación del racismo es importante mencionar, por último, que la negación del racismo tiene tanto una dimensión individual como una dimensión social. A los hablantes no sólo les preocupa parecer racistas ellos mismos, sino que también tienen interés en defender la imagen de su endogrupo, dando a entender que "no somos racistas", o "no somos una sociedad racista” (van Dijk, 1992, p. 89). La relevancia de este punto para el presente artículo radica en que un liceo está constituido por una comunidad escolar, que por definición contiene una dimensión social. Como se verá más adelante, en las entrevistas y focusgroups fueron sobre todo los adultos de dicha comunidad quienes mostraron una preocupación especial por enfatizar que su liceo no es un lugar donde exista racismo.

Finalmente, un tercer concepto relevante para este estudio es la educación anti-racista, que puede ser definida como una iniciativa pedagógica orientada a transmitir conocimientos y habilidades para combatir el racismo, la xenofobia y la violencia. El concepto surgió en el ámbito anglosajón e inicialmente tuvo como foco el racismo estructural del cual son víctimas las personas afrodescendientes. Sobre todo en sus inicios, los representantes de la educación anti-racista levantaron fuertes críticas a la educación multicultural, siendo una de las críticas centrales que el racismo fuera presentado como producto de la ignorancia y los prejuicios individuales, en vez de poner en el centro las relaciones de poder y lo político en general. La educación anti-racista pone énfasis en que las escuelas y salas de clases no son espacios neutrales o a-políticos, y que por lo tanto cualquier forma de enseñanza anti-racista debe entenderse como educación política (Auernheimer, 2003, pp. 150-153). El hecho de que cada forma de racismo sea funcional al tiempo y espacio en que se manifiesta, tiene por consecuencia que, del mismo modo, la educación anti-racista debe ser pertinente a cada manifestación particular de racismo. Al margen de los contenidos específicos de cada forma particular de educación anti-racista, los representantes de este tipo de educación enfatizan en la importancia que tiene 
crear, entre los alumnos, una atmósfera de confianza y aceptación. En un primer momento es necesario dar espacio a las expectativas, los miedos y las preocupaciones de los alumnos, para posibilitar que compartan sus prejuicios y estereotipos. De este modo se facilitaría que luego los alumnos estén abiertos al aprendizaje y a escuchar argumentos nuevos (Kampmann 1999, citada en: Auernheimer, 2003, p. 156).

En cuanto a los aspectos metodológicos del presente artículo, el trabajo de campo se realizó entre marzo y octubre de 2015 en un liceo de enseñanza media (secundaria) de una comuna ubicado en la Región Metropolitana de Chile. Se inició con una entrevista individual a la persona encargada de la dirección del liceo, y posteriormente, a través de la técnica del focusgroup, fueron recogidas las visiones de un grupo de profesores, del equipo directivo (subdirector, inspectores, jefe UTP), de un grupo de alumnos chilenos y de un grupo de paradocentes/ asistentes de la educación. Intentamos asimismo realizar un focusgroup con alumnos haitianos, pero debido a la falta de una lengua compartida éste tuvo que ser suspendido a los pocos minutos de ser iniciado: de los 12 alumnos participantes sólo 2 tenían un nivel de conocimiento del castellano suficiente como para comunicarse, y la investigadora a cargo del guiar esa conversación no habla creole ni suficiente francés como para haber empleado alguna de estas dos lenguas en la reunión. Optamos entonces por elaborar un breve cuestionario escrito que fue traducido del castellano al creole, y luego las respuestas dadas por los alumnos, en creole, fueron traducidas de vuelta al castellano. Durante el trabajo de campo nos enteramos, por un lado, de que hay una persona del equipo directivo que ha asumido la tarea de dar la bienvenida y orientación inicial a los alumnos extranjeros que llegan al establecimiento, y por el otro, que hay un curso específico en el cual están concentrados casi todos los alumnos haitianos de ese nivel de enseñanza media. Bajo el supuesto de que tanto la persona que da la bienvenida y orientación inicial comola persona a cargo de la jefaturadel curso mencionado tendrían una experiencia particular y diferente a la de los otros miembros de la comunidad escolar, se llevaron a cabo entrevistas individuales con dichas personas, aunque anteriormente ya habían participado en alguno de los focusgroups realizados en el establecimiento. En resumen, se realizaron 3 entrevistas individuales, 4 focusgroups, y se aplicó 1 cuestionario a ser respondido por escrito. Participaron en este levantamiento de información un total de 47 personas pertenecientes a la comunidad educativa del liceo en cuestión. Con el fin de salvaguardar el anonimato de las personas que fueron entrevistadas de manera individual, hemos optado por referirnos a dichas personas de manera genérica, independientemente de si dichos entrevistados fueron hombres o mujeres.

\section{Análisis del material recopilado: racismo y negación}

En el siguiente apartado se presentan, en primer lugar, diferentes situaciones que denotan visiones, expresiones verbales o prácticas racistas, y en segundo lugar, de qué maneras la comunidad escolar ha estado enfrentando dichas situaciones. 


\title{
Situaciones de racismo identificadas
}

A través del análisis fue posible identificar cinco situaciones o episodios ocurridos en el liceo y que denotan racismo por parte de diferentes actores de la comunidad educativa. Dichas situaciones fueron tanto de tipo discursivo/ verbal como actitudes o acciones concretas.

Un primer tipo de situación que denota racismo refiere a expresiones racistas a nivel discursivo cuando se está hablando de los alumnos haitianos, pero no en presencia de ellos. Las citas dan cuenta tanto de un racismo biológico (uso del concepto "asco") hasta uno cultural, reflejado en la idea de que "no saben comportarse", porque "no piden perdón”, o porque "son bulliciosos, escandalosos":

\begin{abstract}
"una vez escuché a un profesor hablar del color de los alumnos, que le complicaba” (Pregunta: ¿qué dijo ese profe?). "Que le complicaban los negros. Pero es un profesor con características muy particulares” (Entrevista a la persona encargada de la dirección del liceo, p. 10).
\end{abstract}

“A mi mamá le dan asco que vengan haitianos al país, porque dice: antes Chile era un país de blancos, ahora es de negros y blancos. Me da asco” (Pregunta: ¿Qué opinas tú de eso?). “A veces tengo tanta rabia con los haitianos, que a veces pienso lo mismo, pero a veces no" (¿Por qué a veces te da rabia con los haitianos?). "Porque igual no tienen nada de respeto, por ejemplo un grupo de haitianos eran 5 , me empujaron la otra vez, yo les dije que tuvieran cuidado, se rieron, deberían pedir perdón, no tienen respeto por nada” (Focus alumnos, mujer, p. 14).

"Yo recuerdo una señora (una apoderada), no me diga no, si yo los conozco, son buenos para hablar, son bulliciosos, son escandalosos” (Pregunta: ¿A quiénes se refería?). A los haitianos, ella no daba espacio porque eran bulliciosos, escandalosos" (Entrevista profesor jefe, p. 8).

Un color que incomoda y formas de ser que se vuelven visibles en la medida en que salen de la normalización esperada, dan cuenta de cómo en el discurso cotidiano se nombra al migrante haitiano.

Una segunda situación es una práctica aparentemente recurrente en el liceo, y que refiere a "bromas" que hacen los alumnos chilenos a los alumnos haitianos en base a las características físicas de estos últimos.

(Pregunta: ese ejemplo que tu diste, cuando hay gente que dice ¡ay, se oscureció!... ¿qué otro tipo de frases típicas han escuchado?) "Muestra los dientes (...) Es que en nuestra sala siempre apagamos la luz, y decimos ¡oh no te veo! ¡Sonríe! Lo molestamos porque lo único que se le veía eran los dientes” (Focus alumnos, mujer, p. 10). 
"habían algunos problemas... los varones, principalmente, molestaban a las niñas (Pregunta: ¿Varones chilenos?). "Sí, compañeros" (Pregunta: ¿Cómo las molestaban?). "Por el pelo (...). Esos niños molestaban a las niñas por el pelo, como ellas venían con su peinado... (Pregunta: ¿En qué consistía el molestar?). "En tocarles el pelo", hacerles tallas en relación a eso” (Pregunta: ¿Qué tallas por ejemplo?). “Es que no recuerdo exactamente, pero eran como bromas... que les había dado la corriente, ese tipo de cosas así "(Entrevista profesor jefe, p. 2-3).

Las bromas de estas características son formas de denostar a seres humanos, amparados en el anonimato y la impunidad. Reírse del otro a partir de aspectos corporales, como si se trataran de marcas indelebles, refuerzan las distinciones y sitúan al grupo discriminado en un lugar de inferioridad, pues no es considerado un igual.

Una tercera situación dice relación con agresiones verbales directamente dirigidas a uno de los alumnos haitianos:

“(el alumno) la tomó de los hombros, la niña comenzó a gritar ¡suéltame negro tal por cual! Ella había manifestado a la inspectora acerca del olor de este alumno, y por eso es que ella lo molestaba” (Entrevista a la persona encargada de la dirección del liceo, p. 11).

Entre las respuestas escritas de los alumnos haitianos destaca una, que eventualmente hace referencia a la misma situación recién citada. Dicho alumno escribió lo siguiente (o, más precisamente, el traductor tradujo lo escrito por el alumno de la siguiente manera): “...se debe exhortar a los alumnos chilenos para que dejen de ser racistas. Para que dejen de decirnos negro culiao (...)" (Documento compilado de respuestas escritas de alumnos haitianos, hombre, p. 8).

Una cuarta situación hace referencia a un episodio que no implicó agresión física, pero que a todas luces es expresión de violencia simbólica ${ }^{6}$. Aparentemente esta situación causó cierto revuelo en el liceo, ya que fue comentada por varios miembros de la comunidad escolar:

“A mí me tocó vivir una situación muy fuerte, entre los alumnos le comenzaron a decir a otra que era hedionda. Las llevamos a la oficina con la inspectora general y lo conversamos, tratamos de ser lo más sutil, entre la niña chilena y la otra niña, que se sentía muy mal, porque me decía ¿por qué me dice que estoy hedionda? Y ahí le explicamos a la niña chilena que no era que estuviera sucia, sino que su cuerpo es diferente, y su ph es diferente a los chilenos, y le pedimos que le pidiera disculpas y tuvimos que trabajar mucho con ello, cómo llegar a ellas para que no se sintiera mal la chica” (Focus paradocentes, mujer, p. 6).

"Yo he escuchado a algunas compañeras que las tratan de hediondas, que les miran las axilas, porque están sudadas, y así, las moles- 
tan, y ellas se enojan (Pregunta: ¿Esa es una forma común de molestarse en el colegio, o es hacia los alumnos inmigrantes?) Inmigrantes, porque ellas no andan abrigadas todo el día, están con su camisa y nada más, porque nosotros en sí andamos todos con chaleco, pero ellas siempre andan con camisa, pero sin polerón” (Focus alumnos chilenos, hombre, p. 8).

"Yo pienso que es por la alimentación que es diferente, es lo mismo que pasa con los hindúes, ellos tienen un olor diferente porque su alimentación es muy diferente a la nuestra, ellos también dicen que sienten un olor que a ellos no les gusta, pero de ser limpios, son limpios. ¡Después del deporte es cuando viene el problema!” (Focus equipo directivo, mujer, p. 13).

En estas citas se observa el uso de un biologicismo crudo para establecer distinciones entre los alumnos haitianos y los nacionales, como si los alumnos chilenos no sudaran o no tuvieran mal olor después de la clase de gimnasia o un partido de fútbol en el patio. Lo complejo es que la diferencia (un olor diferente) se instala en el discurso de los profesores y legitima las bromas y acusaciones que realizan los alumnos chilenos.

Finalmente, una quinta situación, mencionada sólo en una entrevista, es una agresión que no sólo fue física, sino que adicionalmente tiene una altísima carga simbólica? .

"tuve una situación la semana pasada, lo informé a inspectoría, orientación, de una niña que estoy segura... yo lo puse en la observación, y que claramente es un acto de discriminación (Pregunta: ¿Qué hace esa niña?) "Le hacía gestos, se burlaba de él, le hacía gestos de mono, y en otro momento se para, y con un encendedor le quema el pelo" (Entrevista profesor jefe, p. 3).

Como fue expuesto más arriba, los alumnos haitianos expresaron algunas de sus opiniones sobre su experiencia en el liceo a través de un breve cuestionario. En él se les preguntó, entre otros aspectos, cuánto tiempo llevaban en Chile, por qué habían decidido matricularse en este liceo en particular, y cómo se habían sentido recibidos por las autoridades, profesores y compañeros del liceo. El cuestionario no contenía ninguna pregunta directamente referida a posibles experiencias de racismo, y sin embargo, 8 de los 15 alumnos que respondieron el cuestionario hicieron referencia a experiencias de racismo. No es posible establecer si las experiencias a las que refieren ocurrieron fuera o dentro del liceo, y exceptuando la cita ya mencionada de uno de los alumnos haitianos, y otra respuesta de una alumna que relata que "los estudiantes me recibieron bien, pero me llamaban negrita”, los alumnos no entregan detalles acerca de las experiencias de racismo que han vivido. Sus comentarios se asemejan bastante entre sí y son del siguiente tenor: "Según mi punto de vista creo que los chilenos deberían dejar de ser racistas porque ellos deben pensar en cómo se sentirían si estuvieran en Haití y nosotros les demostráramos racismo” (mujer, 16 
años), "los chilenos deberían dejar de practicar el racismo" (hombre, 14 años), "Yo quisiera que los alumnos chilenos dejaran de ser racistas, porque todos somos iguales - solamente ellos son rojos" (nota: aquí el traductor del documento añadió el siguiente comentario: es una manera de identificar el color de la piel) (mujer, 15 años), "los alumnos no me recibieron muy bien porque no quieren a los negros y a veces nos insultan (...) Quiero que mis compañeros de clase dejen de ser racistas, no nos entienden, nos insultan, y no quieren hablar con nosotros; quiero que se cambie eso" (mujer, 17 años), "Yo quisiera que los chilenos fueran menos racistas y que no nos miren sobre apariencia, porque a pesar de la diferencia de color, todos somos gente” (mujer, 16 años).

\section{Negación del racismo al interior del liceo}

Junto a la identificación de los diferentes discursos o actos racistas presentados, un segundo resultado que emerge del análisis de las entrevistas y focusgroups es la negación generalizada del racismo al interior del liceo. Resulta interesante llamar la atención sobre el hecho de que todas las citas que de una u otra manera niegan el racismo pertenecen a las personas adultas de la comunidad educativa ${ }^{8}$, y en reiteradas ocasiones dichas citas acompañaron directamente los relatos sobre situaciones de connotación racista. Además, es relevante comentar que la mayoría de las citas refieren a la dimensión social más que a la dimensión individual de la negación del racismo. En este punto, cabe comentar que la negación del racismo al interior de un establecimiento educativo tiene características especiales, porque el liceo es una institución a la cual se le ha encomendado la tarea de formar a los jóvenes que asisten a ella. Probablemente tanto los profesores como los paradocentes y los integrantes del equipo directivo - todos quienes cumplen un rol de modelo de los alumnos- se sienten, al menos hasta cierto punto, responsables de lo que dicen o hacen los alumnos de dicho establecimiento: si reconocieran que los alumnos tienen discursos o actitudes racistas, eso afectaría la evaluación que un observador externo podría hacer sobre el propio racismo de los adultos responsables de la formación, o sobre su capacidad de ponerle freno al racismo dentro del establecimiento.

Hacia el final de este apartado veremos que algunos, pocos, miembros de la comunidad educativa tienen conciencia, y hacen un cierto reconocimiento, de que existe racismo en el liceo. Sin embargo, lo que prevalece es la negación.

\section{Negación directa}

Este primer tipo de negación se caracteriza por el uso de palabras como "jamás" (ha habido discriminación o denuncias por racismo), o bien, enfáticas expresiones de auto-afirmación acerca de un trabajo bien hecho en términos de acoger a todos los alumnos:

"la verdad es que tú vas a la sala o los ves en el patio, por lo demás siempre juntos todos ellos, pero pasándolo muy bien. Pasándolo 
muy bien. Entonces jamás ha venido una apoderada... y también nosotros revisamos mucho este tema todos los meses, en reunión de apoderados... a decirme sabe, a mi hija la saco, porque a mi hija aquí le hacen bullying. Jamás. Jamás” (Entrevista a la persona encargada de la dirección del liceo, p. 6).

“como los aceptan es fantástico, los aceptan muy bien; yo jamás los he escuchado, alguna palabra que vaya en desmedro del niño, a discriminarlo, eso no se da” (Focus equipo directivo, hombre, p. 11).

“...hay estudios que dicen que los chilenos somos clasistas y racistas; yo, desde mi punto de vista, no lo veo así. Nosotros hemos acogido súper bien a los peruanos, colombianos, ecuatorianos, dominicanos, brasileño. Es una característica de nosotros yo creo, es como la firma de nosotros” (Focus paradocentes, mujer, p. 7).

\section{Negación de una intencionalidad racista}

Probablemente el mejor ejemplo de una negación de intencionalidad racista está dado por lo que cotidianamente se califica como "broma". El sentido común indica que una broma es graciosa para todos los presentes; sin embargo, cuando una supuesta broma se hace a expensas de las características corporales de una o más personas, es evidente que deja de ser una situación divertida para quienes detentan esas características. Junto a las "bromas" ya mencionadas como "se oscureció" y "muestra los dientes”, se expuso también el caso de los alumnos chilenos que molestaban a sus compañeras haitianas "por el pelo”. Aquí es relevante mencionar que el profesor que relató dicha experiencia además de plantear que “...eran como bromas... que les había dado la corriente, ese tipo de cosas así”, agregó a continuación que podía entender (ese tipo de humor) y que por lo demás había sido algo puntual:

"Para mí es muy entendible, pero sabía que podía afectar la autoestima de las niñas. Pero más de eso no pasó” (Entrevista a un profesor jefe, p.3).

Otro caso donde se observa la negación de una intencionalidad racista está dada por la siguiente cita, que corresponde a la respuesta de la persona entrevistada frente a la consulta de cómo calificar la situación en que una alumna chilena con un encendedor le quema el pelo a un alumno haitiano. La persona entrevistada argumenta que el uso de encendedores para molestarse entre los alumnos es algo habitual y que por lo tanto no se debe interpretar ese hecho como una agresión racista:

"eso no es novedad, porque entre ellos se han tratado de quemar (...) los alumnos con un encendedor han tratado de quemarse la cara así jugando, los spray. Todas esas cuestiones están prohibidas aquí (...) una vez quemaron un pedazo de banco (...) ahora, lo del pelo me preocupa... ahora, eso pasa con cualquiera” (Entrevista a la persona encargada de la subdirección, p. 9). 


\section{Mitigación: minimización, eufemismos y justificaciones}

Una segunda manera de negar las situaciones de racismo en el establecimiento se caracteriza por la mitigación de esos hechos. Dentro de esta estrategia es habitual la minimización, representada por frases del tipo "fue una situación aislada”, "tuvimos un solo problema” o "hay una niña que es racista":

"Yo me acuerdo cuando llegó la primera niña de Haití (...) ella tuvo problemas, aquí no se veían muchos niños de color en la comuna, yo creo que ella fue una de las primeras. Al principio hubo rechazo, le escribían cosa en las paredes, pero la chica fue muy valiente" (Focus equipo directivo, mujer, p. 4)

“con los inmigrantes, tuvimos un solo problema (...). Los migrantes acá están felices, porque todos los recibimos bien, hasta los mismos alumnos; no hay ningún problema, sólo ese caso puntual, pero aquí los inmigrantes en sí, todo bien”(Focus paradocentes, mujer, p. 3).

"fue una situación aislada que se dio en un curso (...). Trabajé con ella el tema de la tolerancia, hay que trabajar ese concepto, qué significa (...) la verdad que la niña lo entendió. Sí, totalmente” (Entrevista a la persona encargada de la dirección del liceo, p. 11).

Una situación similar se dio en referencia al profesor que manifestó que "le complican los negros”. En la entrevista, la conversación continuó de la siguiente manera:

(Pregunta: ¿Tú dirías que es más bien excepcional?) "Muy excepcional, es más, si alguien lo ha denunciado, jamás ha sido ante la comunidad docente, nunca ha llegado un alumno acá a decirme, ¿̇sabe qué?, - porque yo voy harto al patio - , hay un profesor que nos trata mal; con ese profesor, nunca" (Entrevista a la persona encargada de la dirección del liceo, p. 10).

Mantener las prácticas racistas como elementos aislados o hechos individuales que no comprometen a la institución impide avanzar en una educación antirracista, puesto que el primer paso es comprender que estas prácticas, por más “individuales” que sean, responden a una ideología mayor que sustenta un sistema en donde determinados sujetos son destinados a ocupar una posición de inferioridad respecto de otros.

Otra forma en la que se expresa la mitigación es a través del uso de eufemismos. Lo anterior se ve claramente representado en la siguiente cita:

"No creo que aquí se practique racismo; sí bullying. Sí hay una molestia constante por ser negros, o tener un acento cantadito. Sí hay hostigamiento por características diferentes. Pero nunca nadie se ha ido de un espacio porque hay un haitiano" (Focus profesores, p. 12). 
Finalmente, las últimas citas relativas a la mitigación como estrategia de negación dan cuenta de un intento de justificar el actuar racista. En el primer caso se trata de un profesor que considera la "falta de costumbre" de convivir con personas negras como una justificación suficiente para no tolerarlos, reconociendo con ello, aunque implícitamente, que él no tiene esa tolerancia:

"los adultos no estamos acostumbrados a ver gente negra, pero los jóvenes son más tolerantes” (Focus profesores, hombre, p. 12).

El segundo caso se relaciona con la agresión verbal, descrita más arriba, de una alumna chilena hacia un alumno haitiano ("isuéltame negro tal por cual!”). En forma seguida a ese relato, la persona entrevistada agregó que esa agresión se daba de manera posterior a que la agresora hubiera reportado a inspectoría que, a su juicio, uno de sus compañeros haitianos tenía mal olor. El cierre de este relato deja abierta la interrogante de si la persona entrevistada considera que el reporte previo atenúa la gravedad del insulto, o si incluso libera a la alumna de la sospecha de racismo.

"Ella (la alumna) había manifestado a la inspectora acerca del olor de este alumno, y por eso es que ella lo molestaba” (Entrevista a la persona encargada de la dirección del liceo, p. 11).

\section{Acusación de racismo revertida: “exageraciones” o “ver racismo donde no lo hay"}

Finalmente, una cuarta estrategia de racismo que fue posible observar en la comunidad escolar fue la de tildar las acusaciones de racismo de exageraciones. En concreto, la persona entrevistada plantea que el maltrato entre los alumnos de ese liceo es algo habitual, por lo que no correspondería calificar como racismo el uso de la palabra "negro":

“... yo creo que a veces son exageradas las cosas (...) a lo mejor pudo ser una situación y la profesora lo tomó como una discriminación, y no estoy segura que sea tan así (...) le dijeron negro... (...) oye hueónculiao, así se tratan ellos en la sala, se dicen cualquier cosa (...) a lo mejor lo de negro no fue tan despectivo, sino sólo por decirle negro no más” (Entrevista a la persona encargada de la subdirección del liceo, p. 8).

Una negación similar a la recién descrita se dio al mencionarle a la persona entrevistada la situación de la alumna que con un encendedor le quemó el pelo a un compañero haitiano. Como fue expuesto más arriba, de acuerdo a dicha persona las agresiones con encendedores son habituales en el liceo. Sin embargo, si el acto de quemar el pelo sucede inmediatamente después de haber imitado a un simio con el fin de ofender a un alumno afrodescendiente, realmente no cabe ninguna duda de que la agresión con el encendedor tuvo una intencionalidad racista. 
Finalmente,y tal como se adelantó al inicio de este apartado, junto a las diversas situaciones de racismo descritas y las diferentes maneras en que la comunidad escolar las niega, también es importante relevar quealgunos miembros de la comunidad escolar sí reconocen que existe discriminación racista en el liceo, aunque sólo en una cita se habla explícitamente de racismo:

"Los alumnos tratan mal a los haitianos. Se burlan de ellos” (Focus profesores, p. 11).

“¡Este es el curso de los negros! Lamentablemente lo he escuchado, y con mucho dolor debo decirlo, de muchos profesores" (Entrevista profesor jefe, p. 9).

"El chileno como que se encuentra dueño de la tierra, y le da más confianza para molestar al extranjero. Eso le da como el motivo, así como ¡tú ándate!, es como un derecho a molestar” (Focus alumnos, hombre, p. 12).

“...no tenemos que matar a alguien para ser racistas (...)” (Focus profesores, mujer, p. 12).

Respecto de estas últimas citas, la frase “el curso de los negros” hace alusión al hecho de que en este liceo se agrupó a gran parte de los alumnos haitianos en un mismo curso, con la idea de que iban a poder apoyarse entre ellos. La literatura nos indica que mantener prácticas donde se agrupa o aísla a los estudiantes en base a la nacionalidad, condición extranjera oracialización, constituye una forma de segregación interna. Aunque este tipo de prácticas muchas veces se llevan a cabo con una buena intención, visibilizan la necesidad de entregar herramientas a los profesores, de modo que sean conscientes de los alcances de sus decisiones: una segregación como la descrita corre el riesgo de convertirse en una práctica institucional de discriminación y racismo. La relevancia de las prácticas de los profesores será discutida con mayor profundidad en lo que sigue.

\section{Discusión}

¿Qué consecuencias tienen el racismo y su negación para una educación anti-racista enfocada en la enseñanza secundaria chilena? Esa es la pregunta que se intentará responder en esta última parte del artículo.

Antes de continuar, es relevante plantear que para formular una política anti-racista aplicable a la enseñanza secundaria chilena en general, aún necesitamos saber más sobre cómo funcionan las diferentes variantes del racismo en el país. Este artículo ha versado específicamente sobre el racismo contra afro-descendientes, que por lo demás se está tematizando sólo recientemente en Chile. Es importante enfatizar entonces en que las conclusiones que de este análisis se puedan extraer no necesariamente son aplicables a cualquier tipo de racismo en Chile. 
Hemos visto que al interior del liceo el racismo no se manifiesta sólo de manera solapada ("le complican los negros") o a través de "bromas" ("muestra los dientes", "te dio la corriente"), sino también de manera abierta y explícita (violencia simbólica, insultos, gestos, agresiones físicas). Por su parte, la negación se da a través de la negación directa (“jamás” se ha discriminado a alguien), la negación de una intencionalidad racista ("bromas”), mitigación (minimización, eufemismos y justificaciones) o a través de la estrategia de calificar una sospecha de racismo como una exageración.

¿Cómo enfrentar esta compleja situación, y por dónde partir? Intentando ofrecer una propuesta concreta y factible, vamos a referirnos en primer lugar al público objetivo (quiénes deberían recibir educación anti-racista), y luego -haciendo uso de los conceptos propuestos por los lineamientos curriculares del Ministerio de Educación- a los Objetivos Fundamentales y Contenidos Mínimos Obligatorios que podría contener este tipo de educación.

Respecto del público objetivo, nos parece que si hubiera que priorizar, los primeros en recibir una educación anti-racista debieran ser los profesores actualmente en ejercicio, lo que le plantea una demanda particular a la educación continua. Como hemos visto, son los adultos del establecimiento los que más niegan el racismo, y siguiendo a van Dijk, eso indica también que es entre ellos donde el racismo está más presente: mientras la crítica al endogrupo suele ser una estrategia característica de los antirracistas, la negación del racismo es, con frecuencia, típica para quienes emiten opiniones racistas (van Dijk, 1992, p. 91).

En efecto, durante las entrevistas y los focusgroups se reconoció por parte de diferentes actores que los adultos de ese liceo son más racistas que los alumnos:

"Si yo comparo la generación de nosotros con la generación actual, son bastante menos discriminatorios, bastante menos racistas de lo que podemos ser nosotros" (Entrevista a la persona encargada de la subdirección, p. 11)

Otra cita, ya expuesta anteriormente, que confirma esta apreciación es la siguiente:

"los adultos no estamos acostumbrados a ver gente negra, pero los jóvenes son más tolerantes” (Focus profesores, hombre, p. 12).

Si bien las citas dan cuenta de la percepción de que los adultos serían más racistas, consideramos que ello no tiene necesariamente sustento empírico, pues discursos y prácticas racistas son transversales a la clase, género, edad y nacionalidad. Con todo, el hecho de que los profesores sean un modelo para los alumnos, es razón suficiente para que sean los destinatarios principales (aunque no exclusivos) de una educación anti-racista. 
Van Dijk (2002, p. 195) destaca la importancia de que en relación al racismo las elites sean las primeras en transformarse, ya que hasta cierto punto actúan como modelos al interior de cada sociedad. Si llevamos esta reflexión a los establecimientos educacionales, donde sin duda los profesores son modelos para sus alumnos, se confirma la importancia de que una educación anti-racista se inicie con los profesores.

Una vez implementado algún tipo de formación continua anti-racista para profesores en ejercicio, debería ponerse énfasis en ofrecer educación anti-racista tanto en la formación inicial docente como en la enseñanza escolar, así como promover que los establecimientos educativos involucren activamente a los apoderados en esta tarea.

Respecto de los “Objetivos Fundamentales”, los entendemos aquí como las convicciones centrales sobre las cuales debe basarse la educación anti-racista, y que deben permear la totalidad de la enseñanza de esta temática. A nuestro parecer, los Objetivos Fundamentales de la educación anti-racista en Chile debieran apuntar al menos a los siguientes dos objetivos:

- Transmitir que el racismo es una ideología, y que las jerarquías sociales en base a rasgos corporales o fenotípicos son una construcción social. Esto significa que es legítimo percibir diferencias entre los seres humanos, pero no lo es presentar un ordenamiento jerárquico basado en las diferencias, tal como lo hace la ideología racista.

- Favorecer que los estudiantes comprendan que el racismo constituye una vulneración grave de los derechos humanos y que no debe ser tolerado en una sociedad que aspira a ser justa y democrática.

Los dos objetivos mencionados surgen al observar la libertad con la que los responsables de los discursos o actos de racismo se manifiestan, indicando probablemente que no consideran necesario auto-censurarse. Aunque no tenemos datos empíricos y representativos para afirmar lo que sigue, esto probablemente se explica a través del hecho de que, en términos generales, en Chile aún existe una alta tolerancia al racismo.

En relación a los Contenidos Mínimos Obligatorios, emergen como relevantes al menos 6 temas, que son presentados y comentados a continuación.

1) La historia de la esclavitud y del colonialismo. La esclavitud se basó en un conjunto de planteamientos completamente arbitrarios, y de los que sabemos que nunca debieron haberse legitimado. Sin embargo, los efectos de esclavitud son tan profundos, que hasta el día de hoy muchas personas creen que se basó en razones objetivas, racionales o atendibles por alguna otra razón. Es importante lograr que los estudiantes incorporen este conocimiento y comiencen a observar las relaciones interétnicas de 
manera crítica y reflexiva. Respecto del colonialismo, es importante transmitir por ejemplo que éste no produjo sólo una dominación de los cuerpos, sino también de las mentes de los colonizados, quienes han asumido como propias las visiones racistas y discriminadoras de los poderes coloniales vigentes o pasados. En América Latina, donde la mayor parte de la población es amerindia, de todas formas se observa un racismo de quienes se consideran más blancos hacia quienes son considerados más morenos o negros (Menéndez, 2002, p. 188), fenómeno que ha sido calificado como “endorracismo” (Molina \& Rodríguez, 2001, p. 226). Al respecto, van Dijk plantea que "Los racismos latinoamericanos son sistemas de dominio étnico-racial cuyas raíces históricas se enclavan en el colonialismo europeo así como en su legitimación, es decir, en la conquista, la explotación y el genocidio de los pueblos indígenas amerindios y en la esclavitud de los africanos, idea incluida en el colonialismo europeo" (van Dijk, 2003, p. 99). Sobre los múltiples efectos del colonialismo han reflexionado ampliamente los representantes de los estudios postcoloniales (Beverley, 2004; Rivera Cusicanqui \& Barragan, 1997; Spivak, 1988; Unesco, 1978), así como sus predecesores (Fanon, 1973).

2) La historia y la historicidad del racismo. Tal como plantea el ya citado documento de propuestas para una política pública anti-racista en Chile, es necesario "comprender que el racismo tiene una raigambre histórica que requiere ser reconocida, discutida y analizada para poder contribuir a la conformación de una sociedad chilena más justa" (Universidad de Chile, 2015, p. 10). Adquirir conocimientos de la historia del racismo, y comprender paralelamente la historicidad de este fenómeno, puede contribuir en primer lugar a entender que el racismo no ha existido siempre - sino que es producto de una época histórica con condiciones económicas y sociopolíticas determinadas-, y que por ende, en segundo lugar, tampoco es imposible erradicarlo o al menos aminorarlo al interior de una sociedad. La idea de que "los chilenos siempre hemos sido racistas" (cita que en efecto fue mencionada en una de las entrevistas) despoja al racismo de su historicidad, y con ello, de la convicción de que se le pueda poder freno. Conocer la historia del racismo es, probablemente, una de las condiciones centrales para ser capaz de identificar la presencia de racismo en las relaciones sociales. Y al respecto coincidimos con Petrova (2001, citada en: Nguyen Vo, 2009) cuando dice que el reconocimiento del racismo es un prerrequisito -aunque no sea al mismo tiempo una garantía- para superarlo.

3) Conceptos relevantes, tales como racismo biológico, institucional, cultural y cotidiano (Barker, 1981; Essed, 1991; Taguieff, 2001). Como hemos visto, la literatura sobre el racismo distingue, entre otros conceptos, entre un viejo racismo (“científico", biológico) y un nuevo racismo (cultural, diferencialista). En términos generales se asume que el racismo en el siglo XXI es preponderantemente de tipo cultural y que hasta cierto punto eso constituye un progreso, porque al menos la integridad física de las personas que son víctimas del racismo ya no se ve tan amenazada como antes. Sin embargo, ¿'es esta distinción aplicable a Chile? Lo que el trabajo de campo de esta investigación ha mostrado es que en Chile conviven, aun 
en el año 2015, un “viejo” y un “nuevo” racismo. Lejos de haber quedado en el pasado, el racismo basado en las diferencias corporales ("negro culiao" en relación al color de la piel, "te dio la corriente” en relación al tipo de pelo y “hedionda”, en relación al olor del cuerpo) co-existe junto a apreciaciones que hablan de un racismo cultural ("son bulliciosos, son escandalosos”, o “ellos también encuentran raro nuestro olor”). Es dable pensar que para que una sociedad transite de un tipo de racismo al otro, donde al menos sea políticamente incorrecto manifestar el racismo abiertamente, es necesario que dicha sociedad emprenda un profundo proceso de auto-educación, sea éste gestado internamente o provocado por presiones externas. Hasta la fecha, la sociedad chilena no se ha enfrentado a ningún tipo de presión realmente gravitante como para que el racismo deje de ser considerado como natural, o como parte inherente de nuestra sociedad.

4) Importancia de un uso crítico y reflexivo del lenguaje, con el fin de evitar la reproducción del racismo. Si recordamos la definición más general de racismo ofrecida en este artículo, que lo entiende como la creencia en la existencia las razas humanas, el solo hecho de usar el concepto de raza de manera irreflexiva puede ser leído como un atisbo de racismo. El uso acrítico del concepto “día de la raza” puede ser señal de que a nivel consciente o inconsciente las personas aún crean que es legítimo o correcto hacer clasificaciones entre seres humanos en base a la "raza".

“Antes era impensado por ejemplo realizar una efeméride, por ejemplo el día de la raza, y si era el día de la raza nosotros hacíamos un diario mural y sería. Nosotros, en vista que hace ya dos años llegaron los chicos nos hemos abocado todos a terreno a armar un día de la raza, que es una semana completa (...)” (Entrevista a la persona encargada de la dirección del liceo, p. 8).

En este misma línea, se ha planteado que "si carecemos de conocimientos sobre el racismo, no sabemos cómo el discurso está implicado en su reproducción diaria” (van Dijk, 2002, p. 192).

5) Causas de la migración a nivel global y latinoamericano. Es relevante que como parte de una educación anti-racista se comprenda que "La migración internacional se produce en un mundo dividido en Estados nación, en el que quedarse en el país de nacimiento sigue considerándose la norma e irse a otro país, la excepción”, y que "por ello suele considerarse que la migración es un problema, algo que hay que controlar e incluso frenar, porque puede traer consigo cambios impredecibles” (Castles, 2000, p. 18). Sin embargo, la consideración de la migración como un problema es sólo una manera de mirar este fenómeno, que por cierto no contribuye a crear oportunidades ni a establecer relaciones de equidad entre nacionales y migrantes. Es importante que se comprenda que la migración es un fenómeno inherente a la historia de la humanidad, y que son relevantes las decisiones que tomen los estados en términos de facilitar u obstaculizar la integración de los migrantes en las sociedades de acogida, así como la convivencia de todos quienes van conformando las sociedades, marcadas 
por una alto dinamismo y cambio. Cabe destacar por ejemplo el caso de Argentina, que en su normativa migratoria vigente considera la migración como un derecho, y atribuye al estado la responsabilidad de garantizar ese derecho. Los elementos mencionados, junto a otros, son relevantes porque si ya los movimientos migratorios internacionales de la segunda mitad del siglo XX han sido particularmente intensos, todo parece indicar que en el siglo XXI la importancia de la migración "será todavía mayor, a medida que la movilidad de la población aumente en volumen y adopte nuevas modalidades" (Castles, 2000, p. 17).

6) Ley chilena anti-discriminación. En base a la idea de que dentro de las políticas antirracistas el derecho tiene un rol decisivo, en tanto medida represiva (Wieviorka, 2009, p. 189), emerge como último contenido relevante en el marco de esta discusión la tematización de la ley 20.609, conocida como ley chilena de anti-discriminación, promulgada en julio de 2012. Su objetivo es "restablecer eficazmente el imperio del derecho toda vez que se cometa un acto de discriminación arbitraria” (artículo $1^{\circ}$ ), y define como discriminación arbitraria "toda distinción, exclusión o restricción [...] en particular cuando se funden en motivos tales como la raza o etnia, la nacionalidad [...]" (artículo $2^{\circ}$ ). Nos encontramos aquí con un primer problema: ¿cómo transmitir que la creencia en las razas humanas es incorrecta, si el mismo parlamento chileno valida esa expresión? Por otro lado, respecto de las maneras de probar que hubo discriminación, la ley plantea que "Serán admitidos todos los medios de prueba obtenidos por medios lícitos que se hubieren ofrecido oportunamente y que sean aptos para producir fe [...]. El tribunal apreciará la prueba conforme a las reglas de la sana crítica” (artículo $10^{\circ}$ ). Sin embargo, ¿es la "sana crítica" de una sociedad que no ha emprendido un profundo proceso de auto-educación respecto del racismo una crítica confiable para un posible denunciante? Nos parece que la ley antidiscriminación es un avance frente a la inexistencia anterior de un cuerpo legal específicamente referido a la discriminación, también de tipo racista. No obstante, parece conveniente una revisión del lenguaje empleado, y que la existencia de la ley se vea acompañada de otros elementos de política anti-racista, tal como la educación de profesores y alumnos.

\section{Reflexiones finales}

El racismo presente en las escuelas, y en la sociedad en términos más amplios, constituye una forma de intolerancia, discriminación, negación e invisibilización que experimentan diversos grupos de personas en las sociedades modernas. Avanzar en una educación democrática supone atender estos aspectos, pues una sociedad más igualitaria se basa en el reconocimiento mutuo entre personas de múltiples lugares, clases sociales, género, edades y nacionalidades. Superar estas desigualdades implica reconocer en primer lugar que éstas existen y que son reproducidas a partir de discursos y prácticas cotidianas. En segundo lugar, tal como se indicó, requiere de un trabajo constante de toma de conciencia sobre esta reproducción, de modo de establecer y consensuar en las comunidades escola- 
res, una política que indique claramente aquello que no es posible de aceptar, como por ejemplo, la violencia física y simbólica dirigida a los estudiantes migrantes, de color, y de ciertas nacionalidades.

Finalmente nos parece relevante comentar que sabemos que aun en sociedades plenamente informadas e ilustradas sobre la historia y los efectos del racismo éste sigue presente, en parte porque tanto a nivel individual como colectivo está muy vinculado a las emociones, las que se escapan de aquel ámbito que una educación anti-racista enfocada en la racionalidad puede alcanzar. Sin embargo, y aunque no existan garantías sobre sus efectos, por todos los elementos revisados en el presente artículo consideramos necesario que la educación anti-racista sea incorporada cuanto antes a la formación de profesores y alumnos en Chile. 


\section{Notas}

${ }^{1}$ Investigación del proyecto Fondecyt Postdoctorado n 3150512, años 2015-2016

${ }^{2}$ http://www.publimetro.cl/nota/cronica/hay-mas-de-cuatro-mil-haitianos-en-chile/ xIQneC!vah7jvdLTakKE/ (acceso: 14.11.2014)

${ }^{3}$ Los datos fueron solicitados por mail, y la respuesta por parte del Ministerio llegó por la misma vía. Expresamos nuestro agradecimiento a la Unidad de Estadísticas, Centro de Estudios, División de Planificación y Presupuesto, Ministerio de Educación

${ }^{4}$ Para un lector novato en la temática del racismo, valga aquí la aclaración de que la biología, y especialmente la genética, por lo menos desde la década de 1980 en adelante ha desechado por completo el concepto de raza para clasificar a los seres humanos (Molina \& Rodríguez, 2001, p. 251; Wieviorka, 1992, pp. 25-26) .

5 Según la autora Susan Arndt(2012), tocarle el pelo a una persona negra en efecto puede ser una práctica racista. Hay matices si se trata de una situación entre amigos/as, pero lo es, evidentemente, cuando es un desconocido quien se aproxima a una persona negra para tocarle el pelo o preguntarle si puede hacerlo. Lo es porque implícitamente se está diciendo: me da curiosidad tu pelo porque no es normal, como el mío (Arndt, 2012, p. 150)

${ }^{6}$ La violencia racista es simbólica cuando afecta la integridad moral de una persona, sin consecuencias para su integridad física, y sin alterar directamente su participación en la vida social, económica o política (Wieviorka, 2009, p. 87).

${ }^{7}$ Nos referimos al hecho de que en el pasado, muchas personas negras fueron linchadas o quemadas como castigo de supuestos delitos que les imputaba la población blanca, como ocurrió por ejemplo en los Estados Unidos en la época de segregación post esclavitud (Wieviorka, 2009, p. 99).

${ }^{8}$ Los alumnos chilenos, en cambio, no parecen haberse sentido responsables del actuar de sus compañeras, también chilenas, que acusaron públicamente y sin sutilezas a una parte de sus compañeras haitianas de oler mal. 


\section{Bibliografía}

Arndt, S. (2012), Die 101 wichtigsten Fragen: Rassismus. Verlag C.H. Beck, München.

Auernheimer, G. (2003), Einführung in die Interkulturelle Pädagogik. Wissenschaftliche Buchgesellschaft, Darmstadt.

Barker, M. (1981), The new racism. Conservatives and the Ideology of the Tribe. Junction Books. Londres.

Beverley, J. (2004), Subalternidad y representación. Iberoamericana [u.a.].,Madrid.

Castillo, C., \& del Castillo, M. (2014), "Paisaje urbano, sostenibilidad e inmigración en Santiago de Chile”. Revista Lider, 24, 145-167.

Castles, S. (2000), “Migración internacional a comienzos del siglo XXI: tendencias y problemasmundiales”. Revista Internacional de Ciencias Sociales, 165 , 17-32

Ceja Cárdenas, I. (2015, Febrero), “Migraciones haitianas en la región andina”. Boletín Del Sistema de Información Sobre Migraciones Andinas, 19, 2-13.

Cortez, A., Loredo, P., Muñoz, C., Rodríguez, M. L., \& Vásquez, M. E. (Eds.) (2004), Niños y Niñas Inmigrantes en Chile: Derechos y realidades. Fundación Anide- Colectivo Sin Fronteras, Santiago.

Edelstein, L. (1999), El chiste y la exclusión: aproximación sociológica a los chistes discriminatorios. En La segregación negada. Cultura y discriminación social (pp. 265-275). Editorial Biblos, Buenos Aires.

Essed, P. (1991),Understanding Everyday Racism. An Interdisciplinary Theory (Vol. 2). SAGE Publications, Inc. Newbury Park.

Fanon, F. (1973), Pielnegra, máscarasblancas. Editorial Abraxas, Buenos Aires.

Geulen, C. (2007), Geschichte des Rassismus. Verlag C.H. Beck, München.

Memmi, A. (2000), “What is Racism?”. En S. Martinot (Trad.), Racism. University of Minesotta Press, Minneapolis-London.

Menéndez, E. L. (2002), La parte negada de la cultura. Relativismo, diferencias y racismo (Vol. 16). Edicions Bellaterra, Barcelona.

Molina, L., \& Rodríguez, V. (2001), “Elementos conceptuales y vocabulario incluidos en los documentos”. En Conferencia mundial contra el racismo, la discriminación racial, la xenofobia y las formas conexas de intole- 
rancia (pp. 209-262). San José: Instituto Interamericano de Derechos Humanos- Fundación Ford.

Mosse, G. L. (2006), Die Geschichte des Rassismus in Europa (3a ed.). Fischer Taschenbuch Verlag, Frankfurt am Main:

NguyenVo, A. (2009), Estrategias de negación del racismo. Un estudio de caso sobre la negación en la prensa deportiva (Màster en Lingüística i Aplicacions Tecnològiques). Universitat Pompeu Fabra, Barcelona. Retrieved from http://repositori.upf.edu/handle/10230/5343

OIM-FLACSO (2004), Encuesta sobre Inmigrantes Haitianos en República Dominicana (p. 170). OIM-FLACSO, Santo Domingo.

Pavez, I. (2012), “Inmigración y racismo: experiencias de la niñez peruana en Santiago de Chile”. Si Somos Americanos. Revista de Estudios Transfronterizos, XII(1), 75-99.

Pérez Cosgaya, T. (2008), “Fronteras imaginarias en América Latina. La experiencia migratoria de haitianos en Chile”. Rumbos, año III(3), 69-82.

Rivera Cusicanqui, S., \& Barragan, R. (Eds.) (1997), Debates post coloniales (1a ed.). Ed. Historias, La Paz.

Spivak, G. C. (1988), “Can the subaltern speak?”. En C. Nelson \& L. Grossberg (Eds.), Marxism and the Interpretation of Culture (pp. 271313). Macmillan Education, Basingstoke.

Stefoni, C., Acosta, E., Gaymer, M., \& Casas-Cordero, F. (2008), Niños y niñas inmigrantes en Santiago de Chile. Entre la integración y la exclusión. OIM- UAH.

Taguieff, P.-A. (2001), The force of prejudice. On racism and its doubles. (H. Melehy, Trad.) (Vol. 13). University of Minesotta Press, MinneapolisLondon.

Tijoux, M. E. (2013), “Las escuelas de la inmigración en la ciudad de Santiago: Elementos para una educación contra el racismo”. Polis, 12(35), 287307.

Unesco (Ed.) (1978), Raza y clase en la sociedad postcolonial. Un estudio sobre las relaciones entre los grupos étnicos en el Caribe de lengua inglesa, Bolivia, Chile y México. Unesco, Paris.

Universidad de Chile (2015), Recomendaciones de Políticas Públicas contra el Racismo en Chile. Departamento de Sociología, Facultad de Ciencias Sociales. Retrieved from http://www.uchile.cl/portal/extension-y-cultura/ vicerrectoria-de-extension-y-comunicaciones/catedra-de-derechos-humanos/ 113362/seminario-racismo-en-chile 
Polis, Revista Latinoamericana, Volumen 14, $N^{\circ}$ 42, 2015

Valenzuela, P., Riveros, K., Palomo, N., Araya, I., Campos, B., Salazar, C., \& Tavie, C. (2014), "Integración laboral de los inmigrantes haitianos, dominicanos y colombianos en Santiago de Chile”. Revista Antropologías Del Sur, 2, 101-120.

Van Dijk, T. (1992), "Discourse and the denial of racism”. Discourse \& Society, 87-118.

Ídem (2002), “Discurso y racismo”. Persona y Sociedad, XVI(3), 191-205.

Ídem (2003), Dominación étnica y racismo discursivo en España y América Latina (1a ed.). Gedisa, Barcelona.

Wieviorka, M. (1992), El espacio del racismo. Ediciones Paidós, Barcelona.

Ídem (2009), El racismo: una introducción (1a ed.). Editorial Gedisa, Barcelona.

Recibido: 15.11.2015

Aceptado: 20.12.2015 